\title{
Successful pregnancy after prenatal diagnosis by NGS for a carrier of complex chromosome rearrangements
}

\author{
Jian $\mathrm{Ou}^{1 \dagger}$, Chuanchun Yang ${ }^{2 \dagger}$, Xiaoli Cui ${ }^{2+}$, Chuan Chen ${ }^{2}$, Suyan Ye ${ }^{3}$, Cai Zhang ${ }^{2}$, Kai Wang ${ }^{2}$, Jianguo Chen ${ }^{2}$, \\ Qin Zhang ${ }^{1}$, Chunfeng Qian', Guangguang Fang ${ }^{3,4^{*}}$ and Wenyong Zhang ${ }^{5,6^{*}}$
}

\begin{abstract}
Background: The study is aimed to provide prediction for fertility risk in the setting of assisted reproduction for a woman with complex chromosomal rearrangements (CCRs).

Methods: We implemented a robust approach, which combined whole-genome low-coverage mate-pair sequencing (WGL-MPS), junction-spanning PCR and preimplantation genetic testing for aneuploidy (PGT-A) method to provide accurate chromosome breakpoint junctional sequences in the embryo selection process in the setting of assisted reproduction for a couple with recurrent abortions due to CCRs.

Result: WGL-MPS was applied to a female carrying CCRs which consisted of 9 breakpoints and 1 cryptic deletion related to fertility risks. Sequencing data provided crucial information for designing junction-spanning PCR and PGT-A process, which was performed on the 11 embryos cultivated. One embryo was considered qualified for transplanting, which carried the exact same CCRs as the female carrier, whose phenotype was normal. The amniotic fluid was also investigated by WGL-MPS and karyotyping at 19 weeks' gestation, which verified the results that the baby carried the same CCRs. A healthy baby was born at 39 weeks' gestation by vaginal delivery.

Conclusion(s): Our study illustrates the WGL-MPS approach combining with junction-spanning PCR and PGT-A is a powerful and practical method in the setting of assisted reproduction for couples with recurrent miscarriage due to chromosomal abnormalities, especially CCRs carriers.
\end{abstract}

Keywords: Complex chromosomal rearrangements, Breakpoints mapping, Whole-genome low-coverage mate-pair sequencing, Preimplantation genetic testing for aneuploidy, Junction-spanning PCR

\section{Background}

Complex chromosomal rearrangements (CCRs) are structural rearrangements involving three or more cytogenetic breakpoints on more than two chromosomes [1, 2]. It has been estimated that $3.5 \%$ of couples with a history of recurrent miscarriage have at least one partner who is a carrier of a chromosomal structural rearrangement [3]. The most frequent of these rearrangements is translocation. Other rearrangements include inversions,

\footnotetext{
*Correspondence: Fgg0315@163.com; zhangwy@sustech.edu.cn Jian Ou, Chuanchun Yang, and Xiaoli Cui these are first coauthors.

${ }^{3}$ Shenzhen Dapeng New District Maternity \& Child Health Hospital Department of Gynecology, Shenzhen, China

${ }^{5}$ Southern University of Science and Technology-CheerLand Institute of Precision Medicine, Shenzhen, China

Full list of author information is available at the end of the article
}

insertions, deletions, duplications, or, rarely, ring chromosomes [4]. The potential risk for chromosome imbalance in the gametes of CCRs carriers is higher than those with simple translocations, and thus contributing to higher risk of recurrent miscarriage [5]. The incidence of spontaneous abortions and abnormal pregnancy outcomes in CCRs families was estimated to be 48.3 and $53.7 \%$, respectively [6]. Almost $18.4 \%$ of all live births from CCRs carriers result in phenotypically abnormal offspring and one-half of all CCRs carriers produce offspring who are also CCRs carriers [6]. Moreover, the higher the complexity of CCRs the higher the risk for unbalanced gamete generation and hence the higher the risk for having an affected offspring [7, 8]. In order to assess the risk faced by CCRs carriers who consider

(c) The Author(s). 2020 Open Access This article is distributed under the terms of the Creative Commons Attribution 4.0 International License (http://creativecommons.org/licenses/by/4.0/), which permits unrestricted use, distribution, and 
pregnancy as accurately as possible, precise characterization of CCRs is of crucial importance.

Several cytogenetic and molecular methods such as Giemsa banding, fluorescence in situ hybridization (FISH), array-comparative genomic hybridization and array painting have been applied to study chromosomal structural changes associated with abnormal phenotypes [9]. However, these techniques lack the precision that is required to define the rearrangement at nucleotide level, may fail in identifying smaller chromosomal duplications and deletions, and are often technically challenging and time consuming to perform [10-12].

In recent years, a robust method for global detection of balanced chromosomal rearrangements by wholegenome low-coverage mate-pair sequencing (WGLMPS) has been developed for detailed investigation of CCRs [13]. The approach can identify nearly all cryptic chromosomal abnormalities or complex rearrangements present in the genome. In addition, it is capable of characterizing translocation breakpoints at the nucleotide level [12-15]. Therefore, this method is of value to provide prenatal genetic counseling for couples with reproductive issues by comprehensively mapping CCRs and providing precise breakpoint sequences for subsequent PGT-A.

\section{Methods}

\section{Case presentation}

A young couple (woman and man 27 and 30 years old, respectively) experienced two consecutive early spontaneous miscarriages. The cause of infertility was unknown. Karyotyping was performed on G-banded metaphase spreads of cultured lymphocytes using conventional methods. The man had normal 46, XY karyotype, while the woman was found to carry complex chromosome rearrangement: a q25q28 fragment of chromosome 4 was inserted into q22 in chromosome 1, and this chromosome 4 was shifted in equilibrium with chromosome 5 . The breaking points were on $4 \mathrm{q} 31.1$ and 1q22, respectively. Her karyotype (Fig. 1) is:

46, XX, $\quad \operatorname{der}(1) \mathrm{t}(1: 4)(\mathrm{p} 22: \mathrm{q} 31.1), \operatorname{der}(4) \operatorname{ins}(5: 4)(\mathrm{q} 22 ;$ q25q28)t(1:4),der(5)ins(5:4).

\section{WGL-MPS analysis and breakpoint validation}

According to the results of karyotyping analysis, there was very little possibility for her to give birth to a normal child through natural pregnancy and she faced with an increased risk for having an affected offspring.

To make sure the exact location of the breakpoint and learn more about the risks of abnormal pregnancy outcomes, WGL-MPS was performed on the woman. Her genomic DNA was extracted from peripheral blood with Qiagen DNA extraction kit and then used to construct a non-size selected mate-pair library [12] and then subjected to 50-bp-end multiplex sequencing by BGISeq-500. After removing reads containing sequencing adapters and low-quality reads, the high-quality pair-end reads were aligned to the NCBI human reference genome (hg19, GRCh37.1) using SOAP2. Only uniquely mapped reads were remained for the subsequent analysis as previously described [13, 15]. The breakpoints were validated by junction-spanning PCR as previously described [9]. The PCR primer pairs were reserved sufficiently.

\section{Preimplantation genetic testing for aneuploidy}

The woman used a long protocol, or a GnRH (Gonadotropin-releasing hormone) antagonist protocol for controlled ovarian hyperstimulation. Oocytes were retrieved 34 to $35 \mathrm{~h}$ after hCG injection and fertilized with intracytoplasmic sperm injection (ICSI). we obtained 20 eggs through two cycles and 15 eggs were successfully fertilized, and 11 eventually developed into blastocysts. The ovarian stimulation, oocyte retrieval and embryos culture were performed as described by Yanagimachi R, et al [16]. The trophectoderm cells from the blastocysts were obtained as described by Jian $\mathrm{Ou}$, et al [17], and rinsed three time with G-MOPS (Vitrolife) medium, and then transferred to RNAse-DNAse-free PCR tubes (Axygen) with the minimum medium. Whole genome amplification (WGA) was performed using a QIAGEN kit. Amplification products were stored at $-20^{\circ} \mathrm{C}$. To avoid contamination, this process should be all handled in a ventilation cabinet. The breakpoints validation was performed on the amplification products with the PCR primer pairs kept previously and only three embryos (including two embryos with 9 breakpoints inherited and one embryo without breakpoints) were kept for further analysis. PGT-A was done by comprehensive chromosomal screening on these three embryos [17]. An embryo was found to be a balanced euploid and transferable. After genetic counseling, the couple decided to go ahead with implantation. The HCG level was tested 14 days after the embryo transfer. Pregnancy was confirmed by fetal heartbeat on ultrasonography. Amniocentesis at 19 weeks' gestation was performed to confirm prenatal diagnosis.

\section{Results}

In this study, we presented a unique case of a woman diagnosed with very complex chromosomal rearrangements whose corresponding breakpoints were precisely identified by WGL-MPS. We used junction-spanning PCR to verify the corresponding breakpoints of the embryos generated during assisted reproduction and further checked for aneuploidy by conventional PGT-A. After careful counseling and obtaining consent from the couple, we transplanted a screened qualified embryo and 


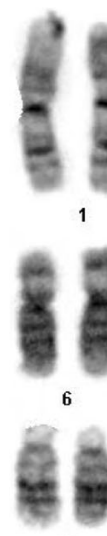

13

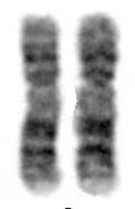

2

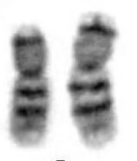

7
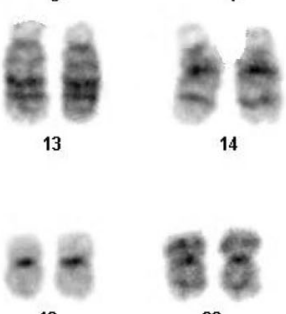

20

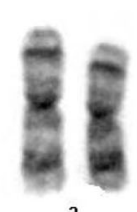

3

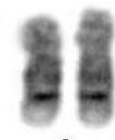

8

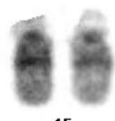

15

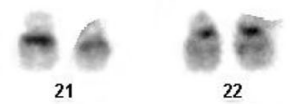

(a) The mother's karyotype

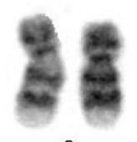

10
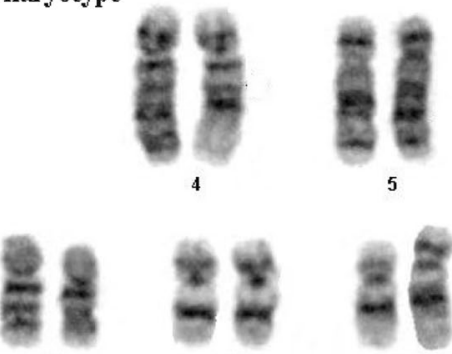

11

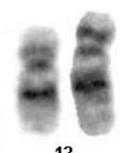

12

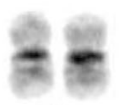

16

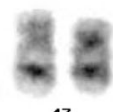

17

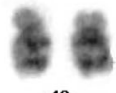

18

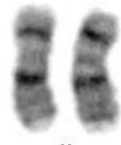

$X \quad Y$

(b) Fetal amniotic fluid karyotype
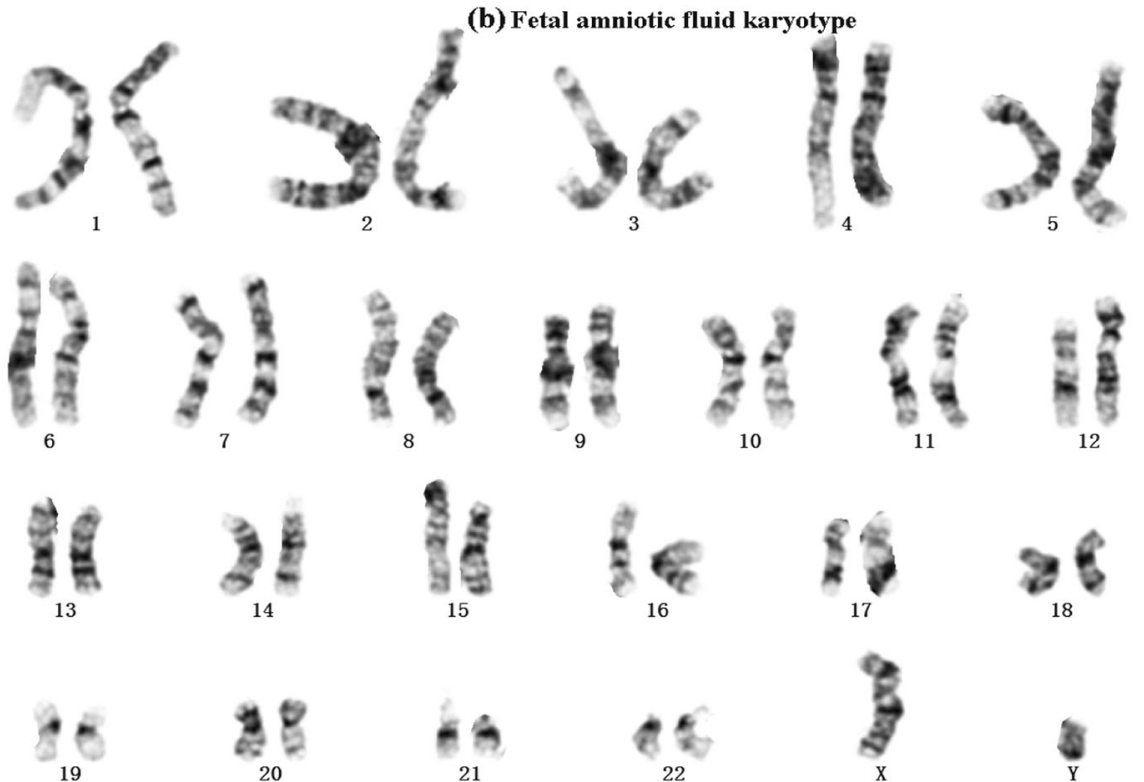

Fig. 1 Maternal and fetal karyotype. (a) Mother karyotype. (b)19-week fetal amniotic fluid karyotype. Their karyotypes are 46, XX, $\operatorname{der}(1) t(1: 4)(p 22: q 31.1), \operatorname{der}(4) \operatorname{ins}(5: 4)(q 22 ; q 25 q 28) t(1: 4), \operatorname{der}(5) \operatorname{ins}(5: 4)$

a normal phenotype baby with the same CCRs as its mother was born. Here we describe such approach (Fig. 2)in the clinical setting.

G-banding analysis at a band resolution of $\sim 400$ revealed the woman to be a carrier of balanced translocation among the three chromosomes and the two breakpoints were on $4 \mathrm{q} 31.1$ and $1 \mathrm{p} 22$, respectively. However, WGLMPS analysis indicated a far more complicated rearrangement. In summary, 9 breakpoints and a microdeletion on chromosome 1 were identified as showed in Fig. 3. Using the new nomenclature for sequenced breakpoints proposed by Ordulu [18], The formula for the chromosome translocation was thus revised as:
46,XX, der(1)ins(1;4)(1qter-> 1p31.1 (5q23.3::1p31.2) $4 \mathrm{q} 28.3->4 \mathrm{qter})$, der(4)t(4:1).

(4pter- > 4q31.1::1p28.3- > 1pter), der(5)ins(5)(5pter- > $5 \mathrm{q} 23.3(\mathrm{t}(4,1)(4 \mathrm{q} 28.3(\operatorname{inv})(1)$

(p31.3::p31.2) inv.(1)(p31.2::p31.1)) 5q23.3- > 5qter).

In our study, four genes, including C1orf141, IL23R, MIER1, SLC35D1 are disrupted at the deletion on 1p31.3. The IL23R gene provides instructions for making a protein called interleukin 23 (IL-23) receptor. Sequence variations in $I L 23 R$ gene have also been associated with the risk of several other immune systemrelated conditions, like psoriasis and inflammatory bowel disease. SLC35D1 is a nucleotide sugar transporter that 


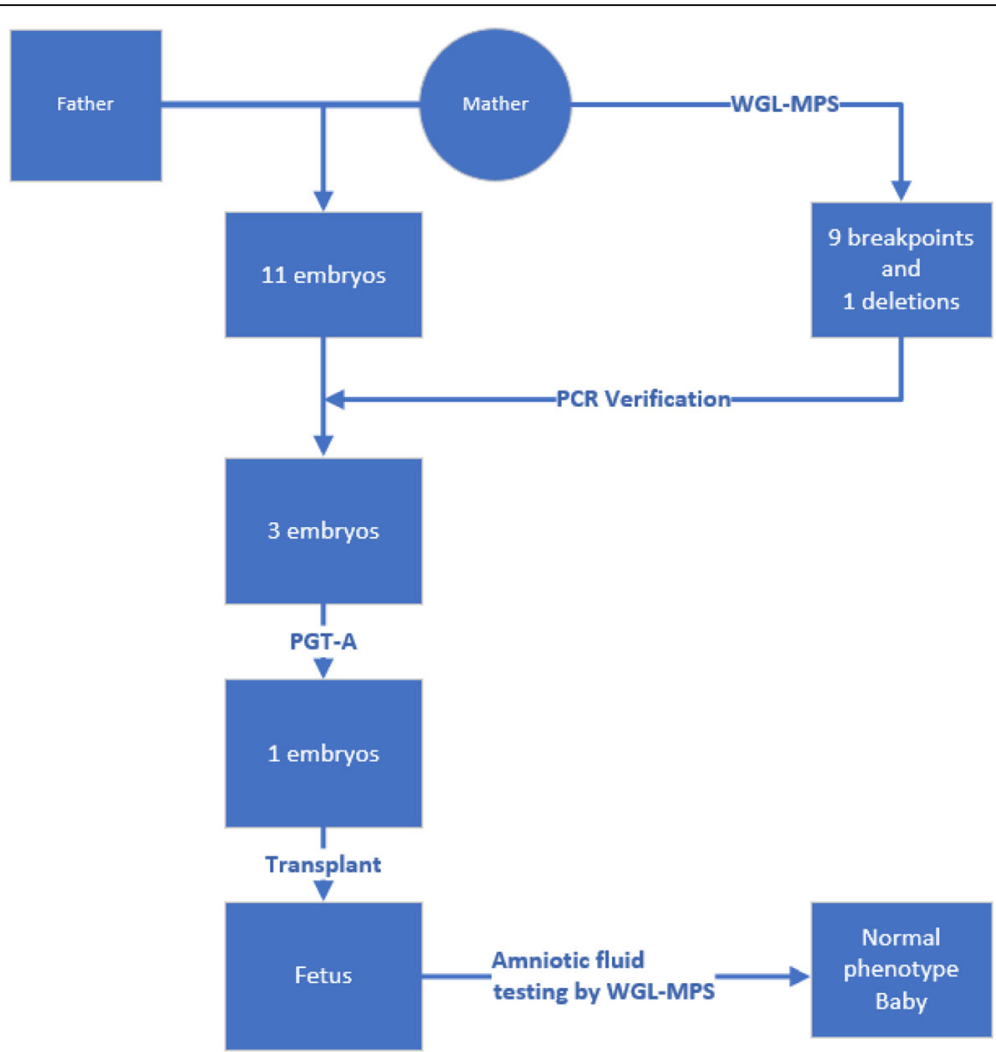

Fig. 2 Experimental operation flow chart. First, we used WGL-MPS technology to detect the CCRs in maternal chromosomes. Secondly, we used PCR to verify the corresponding breakpoints of the 11 embryos generated by serial oocyte verification. Third, we performed PGT-A testing on the selected 3 embryos, and finally obtained an embryo with the same CCRs as the mother. Finally, we transplanted a screened qualified embryo and a normal phenotype baby with the same CCRs as its mother was born

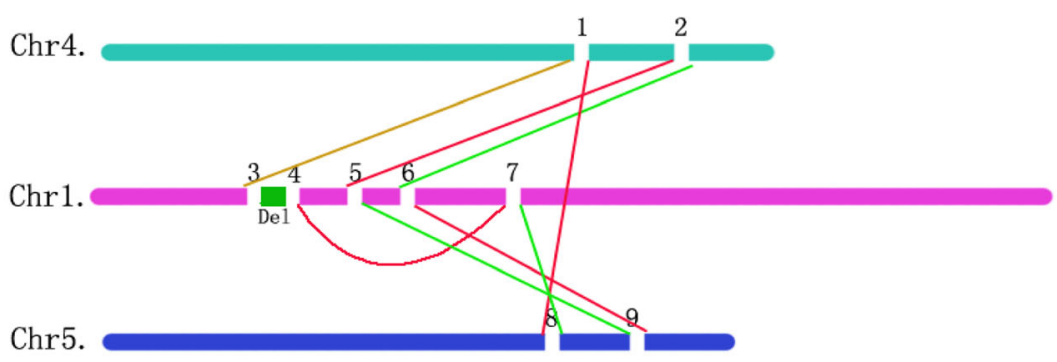

Breakpoint:

chr1. 367416640

chr4. $1134333296 \quad$ chr1.4 67632214

chr5. 8127939569

chr4. 2139154639

chr1. 569057822

chr5. 9129698237

chr1. 669473476

chr1. 784663140

Fig. 3 Reassembly of all chromosomal regions that were involved in the translocations, according to HG19 (www.genome.ucsc.edu) 
localizes to the endoplasmic reticulum and transports both UDP-glucuronic acid and UDP-N-acetyl galactosamine. Homozygous and compound heterozygous lossof-function SLC35D1 mutations have been reported in patients with Schneckenbecken dysplasia. On chromosome 1, the PRKACB gene encoding a catalytic subunit of cAMP-dependent protein kinase (PKA) is interrupted at the 7th breakpoint. On chromosome 4, the SLC7A11 gene is disrupted at the 2nd breakpoint. On chromosome 5, FBN2 and SLC27A6 are disrupted at the 8th breakpoint. The $F B N 2$ gene, encoding a large protein called fibrillin-2, is annotated in OMIM to be associated with autosomal dominant congenital contractual arachnodactyly and early-onset macular degeneration. Fortunately, the woman is not affected by the 8th breakpoint, probably because the breakpoint is close to the end of FBN2 gene sequence. No other known gene is interrupted by the remaining breakpoints, which are breakpoint 1, breakpoint 5, breakpoint 6 and breakpoint 9 .

Eight pairs of primers were designed according to flanking sequences of the breakpoints. The sequences of the primers were displayed in Table 1. If the breakpoints location and sequences were predicted correctly as showed in Fig. 3 and the primers were valid, the corresponding bands of the amplification products should be presented on the electropherogram.

The WGA product of trophectoderm cells from eleven embryos underwent breakpoint analysis using PCR primer pairs designed to amplify junctional sequences and three embryos (including two embryos with 9 breakpoints inherited and one embryo without breakpoints) underwent the PGT-A protocol. PGT-A showed that Embryo4 was chr16 triploid and Embryo9 had a 6q16.1 (93,100,000-99,500,000) deletion (Table 2). A single euploid embryo, identified to carry all the same nine breakpoints as its mother was implanted. Prenatal diagnosis by amniocentesis and WGL-MPS was performed at 19 weeks' gestation, which revealed the fetus to be a carrier of the same complex chromosomal rearrangements and deletion as the mother. A healthy $2780 \mathrm{~g}$ baby was delivered at 39 weeks' gestation by vaginal delivery.

\section{Discussion}

It has previously been demonstrated that precise characterization of apparently balanced CCRs in nonaffected individuals is crucial as they are likely to produce gametes with unbalanced products because of quadrivalent formations during meiosis, which usually results in reproductive failure, recurrent miscarriages or affected offspring [20, 21].

In this study, we present a rare case of a non-affected female experienced recurrent miscarriage with CCRs. The karyotyping report indicates a balanced translation between chromosome 1 and chromosome 4 and a q25q28 fragment of chromosome 4 inserted into chromosome 5q22. However, WGL-MPS utilized in this study allowed accurate reconstruction of the derivative chromosomes, and interestingly revealed a far more complex rearrangement picture compromising translocation of three fragments of chromosome 1, a fragment of chromosome 4 and a fragment of chromosome 5 . It has previously been demonstrated that cryptic deletions are a common finding in "balanced" reciprocal and

Table 1 Primer information of the breakpoints

\begin{tabular}{|c|c|c|c|c|c|c|}
\hline Primer Name & Primer pair & Sequence $\left(5^{\prime}->3^{\prime}\right)$ & Length & Tm & GC\% & Product length [19] \\
\hline \multirow[t]{2}{*}{ P1 } & Forward primer & GGCTGGGAAGTCCAACACGA & 20 & 62.39 & 60 & 382 \\
\hline & Reverse primer & CTAGTTCAGTCTGGATGTGGTCC & 23 & 60.12 & 52.17 & \\
\hline \multirow[t]{2}{*}{ P2 } & Forward primer & GGCAACCTAATCAAGTACGGAA & 22 & 58.4 & 45.45 & 471 \\
\hline & Reverse primer & СTCTCTTGCCTCACAAATGCAC & 22 & 60.1 & 50 & \\
\hline \multirow[t]{2}{*}{ P3 } & Forward primer & GGGAAGAGCCTTGCTCGTA & 19 & 59.1 & 57.89 & 336 \\
\hline & Reverse primer & TAAAGCAGGTATGCGTGAGATTG & 23 & 59.13 & 43.48 & \\
\hline \multirow[t]{2}{*}{ P4 } & Forward primer & GACAAAATGACAGCAATAAGCCC & 23 & 58.82 & 43.48 & 320 \\
\hline & Reverse primer & AGTTGGAAATCCTTCCTCAACTC & 23 & 58.34 & 43.48 & \\
\hline \multirow[t]{2}{*}{ P5 } & Forward primer & TGCAGGTTAAGTCCTCCGTTT & 21 & 59.58 & 47.62 & 421 \\
\hline & Reverse primer & GGGTTATGTTACCCTTCTGCCTAA & 24 & 60.08 & 45.83 & \\
\hline \multirow[t]{2}{*}{ P6 } & Forward primer & TGTAGTGGCACGATCTCAGC & 20 & 59.83 & 55 & 351 \\
\hline & Reverse primer & TCCTTGCCTCTTCCATTTGT & 20 & 57.02 & 45 & \\
\hline \multirow[t]{2}{*}{ P7 } & Forward primer & GCATGGCTCATCATATCGCATAA & 23 & 59.38 & 43.48 & 473 \\
\hline & Reverse primer & TGATGGTGCAACTAATGGCAGA & 22 & 60.29 & 45.45 & \\
\hline \multirow[t]{2}{*}{ P8 } & Forward primer & CTGGCTCAACTITTGATGAGTGT & 23 & 59.43 & 43.48 & 391 \\
\hline & Reverse primer & TTCCAGAGAGTGGGGTCATCT & 21 & 59.92 & 52.38 & \\
\hline
\end{tabular}


Table 2 Embryo screening results

\begin{tabular}{lllllllllll}
\hline Code & Break(1-3) & Break(2-6) & Break(5-9) & Break(8-7) & Break(8-1) & Break(2-5) & Break(4-7) & Break(6-9) & PGT-A result & Result \\
\hline Embryo1 & $\sqrt{ }$ & $\sqrt{ }$ & $\sqrt{ }$ & $\sqrt{ }$ & $\sqrt{ }$ & $\sqrt{ }$ & $\sqrt{ }$ & $\sqrt{ }$ & normal & OK \\
Embryo2 & $\times$ & $\sqrt{ }$ & $\sqrt{ }$ & $\sqrt{ }$ & $\sqrt{ }$ & $\sqrt{ }$ & $\sqrt{ }$ & $\times$ & NA & Failed \\
Embryo3 & $\times$ & $\times$ & $\sqrt{ }$ & $\sqrt{ }$ & $\sqrt{ }$ & $\sqrt{ }$ & $\sqrt{ }$ & $\sqrt{ }$ & NA & Failed \\
Embryo4 & $\sqrt{ }$ & $\sqrt{ }$ & $\sqrt{ }$ & $\sqrt{ }$ & $\sqrt{ }$ & $\sqrt{ }$ & $\sqrt{ }$ & $\sqrt{ }$ & chr16 triploid & Failed \\
Embryo5 & $\times$ & $\times$ & $\sqrt{ }$ & $\sqrt{ }$ & $\sqrt{ }$ & $\sqrt{ }$ & $\sqrt{ }$ & $\sqrt{ }$ & NA & Failed \\
Embryo6 & $\sqrt{ }$ & $\times$ & $\sqrt{ }$ & $\times$ & $\times$ & $\sqrt{ }$ & $\sqrt{ }$ & $\sqrt{ }$ & NA & Failed \\
Embryo7 & $\times$ & $\times$ & $\sqrt{ }$ & $\sqrt{ }$ & $\sqrt{ }$ & $\sqrt{ }$ & $\times$ & $\sqrt{ }$ & NA & Failed \\
Embryo8 & $\times$ & $\times$ & $\sqrt{ }$ & $\sqrt{ }$ & $\sqrt{ }$ & $\sqrt{ }$ & $\sqrt{ }$ & $\sqrt{ }$ & NA & Failed \\
Embryo9 & $\times$ & $\times$ & $\times$ & $\times$ & $\times$ & $\times$ & $\times$ & $\times$ & $6 q 16.1$ del & Failed \\
Embryo10 & $\sqrt{ }$ & $\times$ & $\sqrt{ }$ & $\times$ & $\times$ & $\sqrt{ }$ & $\sqrt{ }$ & $\sqrt{ }$ & NA & Failed \\
Embryo11 & $\times$ & $\sqrt{ }$ & $\sqrt{ }$ & $\sqrt{ }$ & $\sqrt{ }$ & $\times$ & $\times$ & $\sqrt{ }$ & NA & Failed \\
\hline
\end{tabular}

complex chromosome rearrangements, which may explain the clinical phenotypes in many cases [20]. The woman in this case carried CCRs and had already experienced two miscarriages. Due to high degree of her CCRs, there was very little possibility for her to give birth to a normal child through natural pregnancy and she faced with an increased risk for having an affected offspring. After consulting with her physicians, the couple decided to go through the assisted reproduction procedure. Because of CCRs, breakpoints need to be accurately determined before transplantation, and embryos that do not have breakpoints or carry breakpoints like the mother need to be kept. The embryos retained in the above screening should be tested by PGT-A to screen out those with abnormal chromosomal structure and number. If this woman and her child reproduce in the future, they need assisted reproduction and do the above corresponding tests to screen for appropriate embryos. Our case demonstrated that WGL-MPS method combining with junction-spanning PCR and PGT-A could be a powerful and practical tool in the process of risk assessment and embryo selection for couples with recurrent miscarriage due to chromosomal abnormalities.

Precise identification of the breakpoints has been one of the most interesting and technically challenging field in cytogenetics for investigating the possible genotype and phenotypic outcomes of carriers of chromosomal rearrangements. Conventional techniques, such as in situ hybridization with fluorescent dye-labelled bacterial artificial chromosome clones and DNA array hybridization combined with chromosome sorting have been adopted to characterize the chromosome breakpoints to the kilobase level [22-25]. However, these techniques are laborious and expensive. In the recent years, massive parallel sequencing has been developed to accurately detect the breakpoints, but this technique is highly dependent on prior knowledge of the affected G-band region. In our study, we developed a practical solution which could rapidly localize the cryptic breakpoints to individual genes, and substantially improve the prediction of the fertility risks and phenotypic outcomes and timely inform antenatal medical care within a time frame that allows for clinical action. In addition, our approach which could precisely identify the breakpoints down to nucleotide level, can better assess the genotypic and phenotypic consequences of chromosomal abnormalities.

\section{Conclusions}

Accurate breakpoints mapping is the key to provide prediction for fertility risk, genetic counseling, and fertility guidance for couples who carry CCRs. In this study, a robust approach, whole-genome low-coverage mate-pair sequencing (WGL-MPS), was applied to a female CCRs carrier without taking advantage of the result of Gbanding, precisely revealed 9 breakpoints and 1 cryptic deletion related to fertility risks, and provided crucial information for the PGT-A process. Junction-spanning PCR and PGT-A were performed on the 11 embryos cultivated and only one embryo was considered qualified which carried the exactly same CCRs as the female carrier, whose phenotype was normal. The amniotic fluid was also investigated by WGL-MPS, which verified the baby carried the same CCRs. A healthy baby was delivered at 39 weeks' gestation by vaginal delivery. Our study illustrates the WGL-MPS approach especially combining with junction-spanning PCR and PGT-A is a valuable tool in assisted reproduction for couples with complex chromosomal abnormalities and recurrent miscarriages.

\section{Acknowledgements}

The authors thank The Affiliated Suzhou Hospital of Nanjing Medical University for providing samples and ethical approval and TechnologyCheerLand Institute of Precision Medicine for providing research equipment.

\section{Authors' contribution}

Jian Ou - made substantial contributions to conception and design, acquisition of data; been involved in drafting the manuscript and given final approval of the version to be published. Chuanchun Yang - made substantial 
contributions to conception and design and modify the manuscript. Xiaoli Cui-made substantial contributions to conception and design, acquisition of data, analysis and interpretation of data; been involved in drafting the manuscript. Chuan Chen and Jianguo Chen-made substantial contributions to biological experiments and acquisition of experimental data. Suyan Yemade substantial contributions to revise the manuscript critically for important intellectual content. Cai Zhang and Kai Wang - investigated and read relevant background documents; been involved in revising the manuscript critically for important intellectual content. Qin Zhang and Chunfeng Qian - acquisition of sample data; been involved in drafting the manuscript. Guangguang Fang made substantial contributions to revise the manuscript critically for important intellectual content and given final approval of the version to be published. Wenyong Zhang made substantial contributions to conception and design; been involved in revising the manuscript critically for important intellectual content and given final approval of the version to be published.

\section{Funding}

This study was supported by the Industrial Technology Innovation Project of Suzhou City (SYS201566), Jiangsu Province "13th Five-Year" Youth Medical Person Program (QNRC2016246) and Special Fund for Science and Technology Innovation and Industrial Development in Dapeng New District, Shenzhen (YL201800201).

\section{Availability of data and materials}

The datasets used and/or analyzed during the current study are available from the corresponding author on reasonable request.

\section{Ethics approval and consent to participate}

All procedures performed in the study involving samples were in accordance with the ethical standards of The Affiliated Suzhou Hospital of Nanjing Medical University, Suzhou Municipal Hospital.

\section{Consent for publication}

Not applicable.

\section{Competing interests}

The authors declare that they have no competing interests.

\section{Author details}

${ }^{1}$ Center for Reproduction and Genetics, The Affiliated Suzhou Hospital of Nanjing Medical University, Suzhou Municipal Hospital, Suzhou 215002, China. ${ }^{2}$ CheerLand Biological Technology Co., Ltd, Shenzhen 518000, China. ${ }^{3}$ Shenzhen Dapeng New District Maternity \& Child Health Hospital Department of Gynecology, Shenzhen, China. ${ }^{4}$ Shenzhen Second People's Hospital, The First Affiliated Hospital of Shenzhen University, Shenzhen, China. ${ }^{5}$ Southern University of Science and Technology-CheerLand Institute of Precision Medicine, Shenzhen, China. ${ }^{6}$ School of Medicine, Southern University of Science and Technology, Shenzhen, China.

\section{Received: 7 November 2019 Accepted: 10 February 2020}

\section{Published online: 29 February 2020}

\section{References}

1. Pai GS. G.H. Thomas, W. Mahoney, and B.R. Migeon, Complex chromosome rearrangements. Report of a new case and literature review. Clin Genet. 1980; 18:436-44.

2. Kleczkowska A, Fryns JP, Van den Berghe H. Complex chromosomal rearrangements (CCR) and their genetic consequences. J Genet Hum. 1982; 30:199-214.

3. Stephenson MD. Frequency of factors associated with habitual abortion in 197 couples. Fertil Steril. 1996;66:24-9.

4. Sierra S, Stephenson M. Genetics of recurrent pregnancy loss. Semin Reprod Med. 2006;24:17-24.

5. Escudero T, Estop A, Fischer J, Munne S. Preimplantation genetic diagnosis for complex chromosome rearrangements. Am J Med Genet A. 2008;146A 1662-9.

6. Gorski JL, Kistenmacher ML, Punnett HH, Zackai EH, Emanuel BS. Reproductive risks for carriers of complex chromosome rearrangements: analysis of 25 families. Am J Med Genet. 1988;29:247-61.
7. Pellestor F, Anahory T, Lefort G, Puechberty J, Liehr T, Hedon B, Sarda P. Complex chromosomal rearrangements: origin and meiotic behavior. Hum Reprod Update. 2011;17:476-94.

8. Madan K. Balanced complex chromosome rearrangements: reproductive aspects. A review. Am J Med Genet A. 2012;158A:947-63.

9. Aristidou C, et al. Accurate breakpoint mapping in apparently balanced translocation families with discordant phenotypes using whole genome mate-pair sequencing. PLoS One. 2017;12:e0169935.

10. Chen W, et al. Breakpoint analysis of balanced chromosome rearrangements by next-generation paired-end sequencing. Eur J Hum Genet. 2010;18:539-43.

11. Le Scouarnec S, Gribble SM. Characterising chromosome rearrangements: recent technical advances in molecular cytogenetics. Heredity (Edinb). 2012; 108:75-85.

12. Luo A, et al. Maternal interchromosomal insertional translocation leading to 1q43-q44 deletion and duplication in two siblings. Mol Cytogenet. 2018;11:24.

13. Dong $Z$, et al. A robust approach for blind detection of balanced chromosomal rearrangements with whole-genome low-coverage sequencing. Hum Mutat. 2014;35:625-36.

14. Yao $\mathrm{H}$, et al. Breakpoints and deleted genes identification of ring chromosome 18 in a Chinese girl by whole-genome low-coverage sequencing: a case report study. BMC Med Genet. 2016;17:49.

15. Li $L$, et al. Mapping breakpoints of a familial chromosome insertion $(18,7)$ (q22.1; q36.2q21.11) to DPP6 and CACNA2D1 genes in an azoospermic male. Gene. 2014;547:43-9.

16. Yanagimachi R. Intracytoplasmic injection of spermatozoa and spermatogenic cells: its biology and applications in humans and animals. Reprod BioMed Online. 2005;10:247-88.

17. Ou J, et al. Identification of small segmental translocations in patients with repeated implantation failure and recurrent miscarriage using next generation sequencing after in vitro fertilization/intracytoplasmic sperm injection. Mol Cytogenet. 2015;8:105.

18. Ordulu $Z$, et al. Describing sequencing results of structural chromosome rearrangements with a suggested next-generation cytogenetic nomenclature. Am J Hum Genet. 2014;94:695-709.

19. Toufaily MH, Roberts DJ, Westgate MN, Holmes LB. Triploidy: variation of phenotype. Am J Clin Pathol. 2016;145:86-95.

20. De Gregori $M$, et al. Cryptic deletions are a common finding in "balanced" reciprocal and complex chromosome rearrangements: a study of 59 patients. J Med Genet. 2007;44:750-62.

21. Aleksandrova $\mathrm{N}$, et al. Comparison of the results of preimplantation genetic screening obtained by a-CGH and NGS methods from the same embryos. Gynecol Endocrinol. 2016;32:1-4.

22. Baronchelli $\mathrm{S}$, et al. Investigating the role of $\mathrm{X}$ chromosome breakpoints in premature ovarian failure. Mol Cytogenet. 2012;5:32.

23. Scott SA, Cohen N, Brandt T, Warburton PE, Edelmann L. Large inverted repeats within Xp11.2 are present at the breakpoints of isodicentric $X$ chromosomes in Turner syndrome. Hum Mol Genet. 2010;19:3383-93.

24. Fonseca AC, Bonaldi A, Bertola DR, Kim CA, Otto PA, Vianna-Morgante AM. The clinical impact of chromosomal rearrangements with breakpoints upstream of the SOX9 gene: two novel de novo balanced translocations associated with acampomelic campomelic dysplasia. BMC Med Genet. 2013; 14:50.

25. Vergult S, et al. Mate pair sequencing for the detection of chromosomal aberrations in patients with intellectual disability and congenital malformations. Eur J Hum Genet. 2014;22:652-9.

\section{Publisher's Note}

Springer Nature remains neutral with regard to jurisdictional claims in published maps and institutional affiliations. 